\title{
Amenorrea Post-gestación
}

\author{
Facultad de Medicina de Valencia \\ Cátedra de Obstetricia y Ginecología \\ Prof. F. Bonilla-Musoles \\ Dres.: E. Acosta Cajiao* , A. Millet Part**, \\ J. Ferrer Barriendos*** y A. Blanes Espi.****
}

\section{INTRODUCCION}

Pocos trabajos recientes se preocupan de la importancia que la gravidez tiene como factor desencadenante de amenorrea secundaria. Si bien la incidencia de Síndrome de Sheehan ha disminuido en los últimos años, probablemente debido a la mejoría en la atención obstétrica y las facilidades de transfusión (4.62), la posibilidad de efectuar determinaciones hormonales muy precisas por medio del radioinmunoanálisis y los nuevos conocimientos acerca de la fisiología de la prolactina han permitido esclarecer la fisiopatología de cuadros de amenorrea que hasta ahora permanecían oscuros, o eran englobados en síndromes con nombres propios como Argonz del Castillo, Chiari Frommel o Forbes Albright.

* Residente de II Año de Ginecología y Obstetricia.

** Jefe del Departamento de Endocrinología Ginecológica.

** Profesor Agregado de la Cátedra de Ginecología y Obstetricia. Jefe del Departamento de Esterilidad Matrimonial.

**** Médico Adjunto al Departamento de Mama.
Para poder valorar su importancia hemos revisado aquellas pacientes que habían acudido a la clínica aquejando amenorrea secundaria a una gestación. El presente trabajo nos ha permitido encontrar varios síndromes con mecanismos fisiopatológicos tan diferentes como la amenorrea hiperprolactinémica, la disfunción hipotalámica, la menopausia precoz, el síndrome de Sheehan y el síndrome de Asherman.

\section{MATERIAL Y METODOS}

Nuestro material está constituido por 40 pacientes que han consultado al departamento de Endocrinología Ginecológica de nuestra clínica por presentar una amenorrea secundaria que se iniciaba a partir de una gestación, bien post-parto, bien post-aborto, y que habian suspendido la lactancia por lo menos 6 meses antes del momento de la consulta.

Para la clasificación de estas pacientes nos basamos en:

1. Historia Clínica cuidadosa, haciendo especial hincapié en: a) la anamnesis menstrual previa a la gestación, descartando sistemáticamente toda enferma que presentase cualquier tipo de altera- 
ción anterior al embarazo; b) el curso de la gestación: evolución del embarazo (normal o patológico) y parto (espontáneo, intervenido o quirúrgico), presencia o ausencia de patología puerperal, lactancia natural o artificial (voluntaria o involuntaria); c) los antecedentes personales de la enferma: investigando su estado anímico habitual, la presencia de cefaleas, la ingestión de medicamentos que puedan interferir con el ciclo (hormonas, tranquilizantes, etc.), o la aparición de sofocos y oleadas de calor; d) los antecedentes familiares: episodios similares de alteración menstrual, edad de inicio de la menopausia, etc.; e) examen físico completo con determinación de peso y talla, búsqueda sistemática de galactorrea a la expresión, e inspección detenida de piel y faneras.

2. Valoración hormonal, para la cual se practicaron sistemáticamente determinaciones radioinmunoanalíticas de FS$\mathrm{H}$ (valores normales de 3 a $15 \mathrm{mu} / \mathrm{ml}$.), $\mathrm{LH}$ (valores normales de 5 a $30 \mathrm{mu} / \mathrm{ml}$.) (45) y Prolactina (P RI) (valores normales de menos de $25 \mathrm{ng} / \mathrm{ml}$.); determinaciones urinarias de 17 cetoesteroides (valores normales $10 \pm 3 \mathrm{mg} / 24 \mathrm{~h}$ ) y 17 hidroxiesteroides (valores normales $7 \pm$ $3 \mathrm{mg} / 24 \mathrm{~h}$ ) por método de Zimmerman, apoyados en ocasiones por test de estimulación-frenaje. En todas las pacientes se realizó test de progesterona $(15 \mathrm{mg} /$ día de medroxiprogesterona durante 5 días-

Por ser, sin embargo, más significativos $y$ fidedignos nos limitaremos en el presente trabajo a correlacionar los distintos grupos diagnósticos con los valores de FSH, LH, y Prolactina.

3. Estudios radiológicos: Todas las pacientes fueron sometidas a radiografía simple de silla turca, practicándose T.A. C. o Neumoencefalograma cuando la placa simple justificaba la indicación. Igualmente se practicó histerosalpingografía en las pacientes cuyo cuadro clínico justificaba esta exploración.

\section{RESULTADOS}

Encontramos 40 casos de amenorreas post gestación entre un total de 363 pacientes que consultaban por amenorrea secundaria, lo que arroja un porcentaje del $11 \%$. Nuestros hallazgos etiológicos fueron los siguientes: amenorrea hiperprolactinémica, 15 casos $(37.5 \%)$; amenorrea hipotalámica, 14 casos $(35 \%)$; menopausia precoz, 6 casos $(15 \%)$; Síndrome de Sheehan, 3 casos $(7.5 \%)$; Síndrome de Asherman, 2 casos $(5 \%)$. (Ver Tabla No. 1).

\section{Amenorreas hiperprolactinémicas \\ (Tabla No. 2).}

El criterio diagnóstico para la hiperprolactinemia fue el hallazgo de valores superiores a $25 \mathrm{ng} / \mathrm{ml}$ en el promedio de 2 o más determinaciones de prolactina; el valor promedio de todos los casos fue de $66.9 \mathrm{ng} / \mathrm{ml}$. El diagnóstico de adenoma se basó en las imágenes radiológicas de la silla turca (71). La distribución fue la siguiente: hiperprolactinemia funcional, 11 casos $(73.3 \%)$; adenoma hipofisario, 3 casos $(20 \%)$; y sospecha de adenoma, 1 caso $(6.6 \%)$.

Las edades de estas pacientes oscilaron entre 22 y 37 años, con un promedio de 29.3. De las 15 pacientes, 8 eran primíparas $(53.3 \%)$ y 7 multíparas $(46.6 \%)$.

En 3 pacientes la amenorrea fue secundaria a abortos legrados $(20 \%)$, en 8 a partos normales $(53.3 \%), 2$ fueron consecutivas a una cesárea $(13.3 \%), 1$ a un parto prolongado con hemorragia y transfusión, y una a un aborto con hemorragia post legrado y transfusión.

Cuatro pacientes amamantaron (26. $6 \%)$, una dio lactancia mixta $(6.6 \%)$ y las 10 restantes artificial voluntaria $(66.6 \%)$.

Todas las pacientes presentaban galactorrea en el momento del examen, espontánea en $14(93.3 \%)$ y en una $(6.6 \%)$ a la expresión. 
Tabla No. 1

\section{DISTRIBUCION DE LOS DIAGNOSTICOS ETIOLOGICOS EN LAS AMENORREAS POST-GESTACION}
Diagnóstico
No. de casos
Porcentaje

\begin{tabular}{|l|c|c|}
\hline Sindrome de Asherman & 2 & $5 \%$ \\
\hline Sindrome de Sheehan & 3 & $7.5 \%$ \\
\hline Menopausia precoz & 6 & $15 \%$ \\
\hline $\begin{array}{l}\text { Amenorreas } \\
\text { hipotalámicas }\end{array}$ & 14 & $35 \%$ \\
\hline $\begin{array}{l}\text { Amenorreas } \\
\text { hiperprolactinémicas }\end{array}$ & 15 & $37.5 \%$ \\
\hline
\end{tabular}

Tabla No. 2

\section{AMENORREAS HIPERPROLACTINEMICAS}

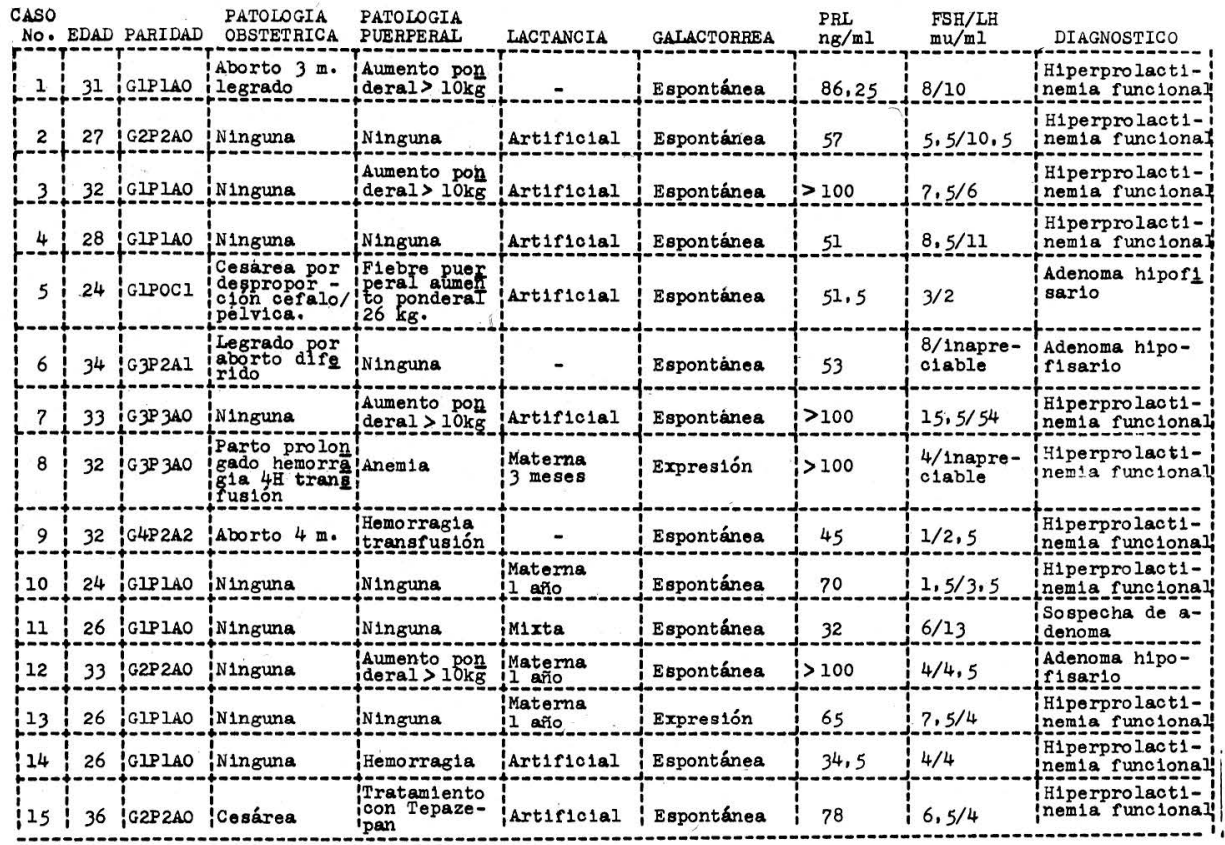


Los valores de gonadotrofinas estuvieron bajos en 4 casos $(26.6 \%)$, selectivamente disminuida la $\mathrm{LH}$ en un caso $(6$. $6 \%$ ) y en los otros 10 los valores fueron normales $(66.6 \%)$.

El tratamiento fue exclusivamente con Bromocriptina en 13 casos $(86.6 \%)$, en un caso $(6.7 \%)$ con resección transesfenoidal $y$ en un caso de hiperprolactinemia iatrogénica inducida por tepazepan se suspendió la medicación.

Los resultados del tratamiento pueden verse en la tabla No. 3.

\section{Amenorreas hipotalámicas Tabla No. 4}

El diagnóstico de amenorrea hipotalámica se basó en la presencia de al me- nos 3 de los siguientes parámetros: disturbios psicológicos identificables con algún componente de depresion, valores de hormonas pituitarias y ováricas dentro de límites normales, variaciones ponderales superiores a $\pm 10 \mathrm{~kg}$., o retorno de reglas cíclicas después de psicoterapia de apoyo y adelgazamiento o tratamiento concomitantes.

Las edades de estas pacientes oscilaron entre 22 y 36 años, con un promedio de 27 . Ocho $(67.1 \%)$ eran multigestantes $y$ seis $(42.9 \%)$ primigestantes.

Doce pacientes $(85.7 \%)$ presentaron patología obstétrica en la gestación inmediatamente anterior a su episodio de amenorrea. Siete pacientes $(50 \%)$ presentaron un aumento de peso superior a

Tabla No. 3

\section{RESULTADOS DEL TRATAMIENTO EN LAS AMENORREAS HIPOTALAMICAS}

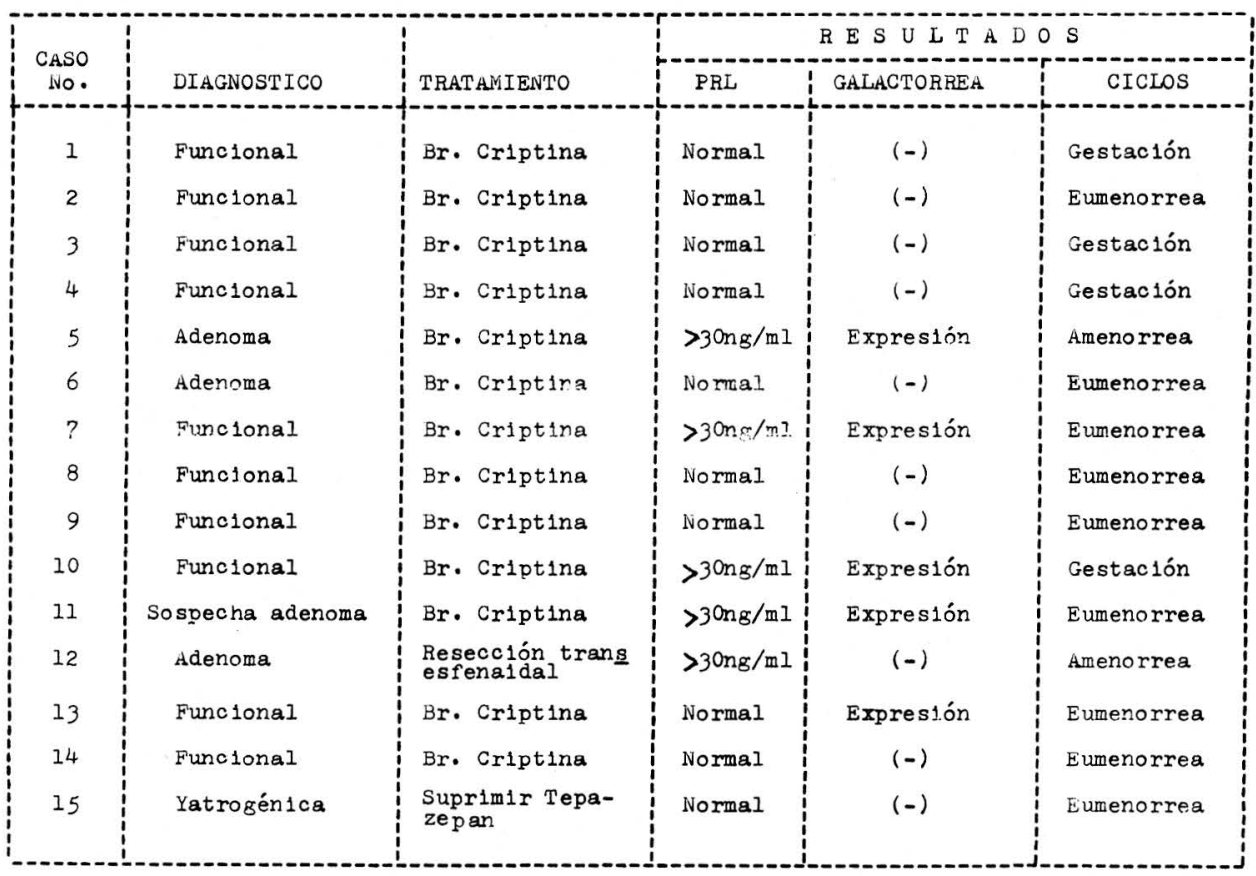


Tabla No. 4

\section{AMENORREAS HIPOTALAMICAS}

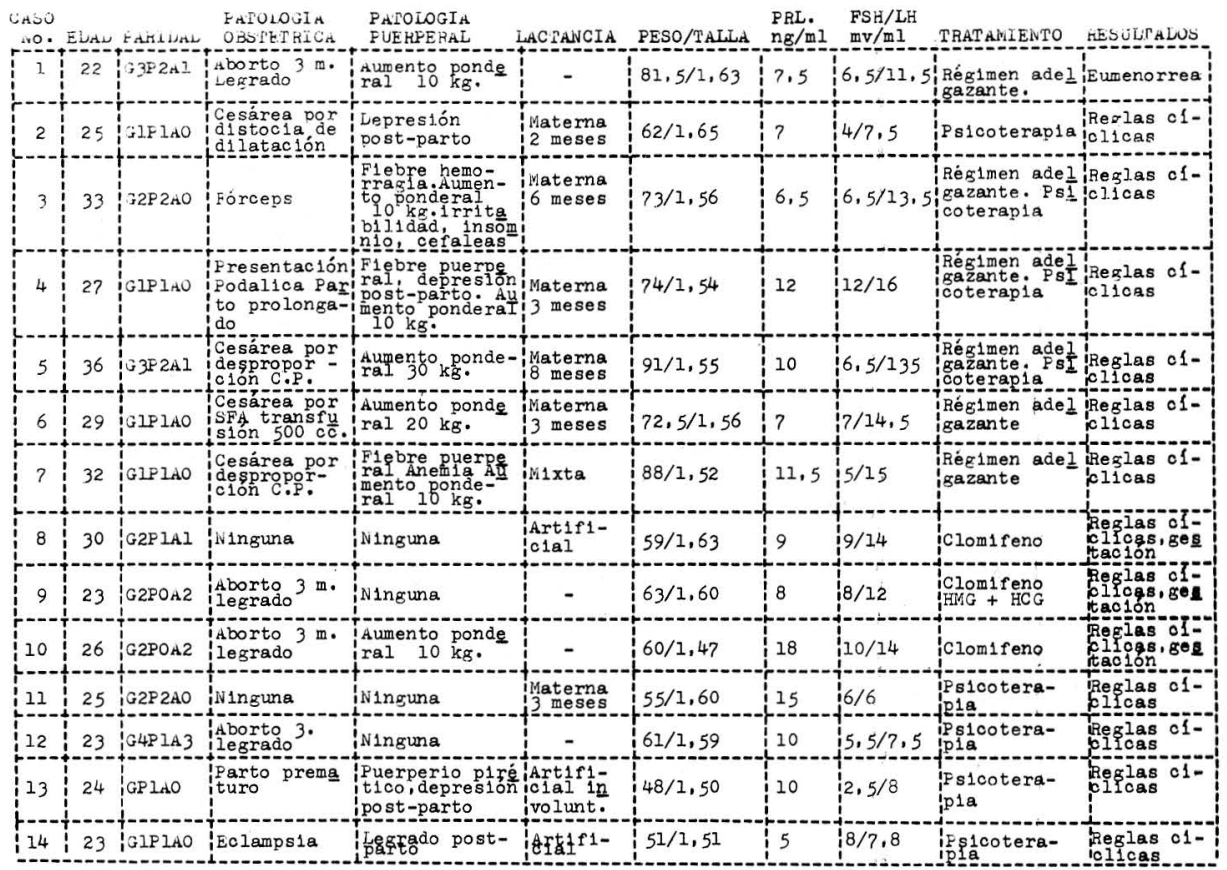

$10 \mathrm{~kg}$. en el período post-parto. Cuatro pacientes $(25.5 \%)$ presentaban alteraciones psíquicas identificables en el puerperio inmediato o tardío. Dos (14. $25 \%$ ) presentaron hemorragia puerperal $y$ cuatro $(28.5 \%)$ tuvieron un puerperio febril.

Ninguna paciente tuvo agalactia. De las 10 pacientes con hijos vivos, $6(60 \%)$ dieron lactancia materna, 3 artificial $(30 \%)$ y una $(10 \%)$ mixta.

Todas las pacientes presentaron valores de PRL, FSH y LH dentro de límites normales.

Once pacientes $(78.57 \%)$ recuperaron sus reglas cíclicas después de psicoterapia y/o régimen adelgazante, dos quedaron gestantes después de tratamiento con Clomifeno $(14.25 \%)$ y una $(7.5 \%)$ después de tratamiento con HMG y HCG.

\section{Menopausia precoz (Tabla No. 5)}

El criterio fundamental para el diagnóstico de menopausia fue el que las pacientes presentaran dos o más determinaciones valores promedio de FSH y LH superiores a 15 y $30 \mathrm{mU} / \mathrm{ml}$ respectivamente. En una paciente el diagnóstico fue comprobado histológicamente, hallándose un ovario rudimentario atrésico.

Aunque la edad en el momento de la consulta osciló entre 33 y 37 años, hay que hacer notar que en el momento del 
Tabla No. 5

\section{MENOPAUSIAS PRECOCES}

\begin{tabular}{|c|c|c|c|c|c|c|c|c|}
\hline $\begin{array}{l}\text { CASO } \\
\text { NO. }\end{array}$ & $\begin{array}{l}\text { EDAD DE } \\
\text { CONSUITA }\end{array}$ & $\begin{array}{l}\text { EDAD UE } \\
\text { IINSTAURACION }\end{array}$ & PARIDAD & $\begin{array}{l}\text { PATOLOGIA } \\
\text { OBSTETRICA }\end{array}$ & $\begin{array}{l}\text { PATOLOGIA } \\
\text { PUERPERAL }\end{array}$ & LACTANCIA & $\begin{array}{l}\mathrm{FSH} / \mathrm{LH} \\
\mathrm{mu} / \mathrm{ml} .\end{array}$ & $\begin{array}{l}\text { PRL } \\
n g / m 1 .\end{array}$ \\
\hline 1 & 34 a. & 27 a. & G2P2 & $\begin{array}{l}\text { Toxem1a } \\
\text { Fdo. Muerto }\end{array}$ & Ninguna & - & $.6 / 85$ & 9.5 \\
\hline 2 & 35 a. & 31 a. & G6P 3 & $\begin{array}{l}\text { Aborto } 40 \\
\text { dlas }\end{array}$ & $\begin{array}{l}\text { Hepatitis. } \\
\text { Sinţomas me } \\
\text { nopausicos. }\end{array}$ & - & $3 / 32$ & - \\
\hline & & $2 ?$ & G2P 2 & Ninguna & nopáusicos & voluntaria & $42 / 61,4$ & 7 \\
\hline 4 & & 318 & G4P 3 & desproporcion & Ninguna & Materna & $18,3 /$ & 8 \\
\hline 5 & & $28 a$ & G2PO & despmoporción & Ninguna & Matams? & & - \\
\hline 6 & a. & 32 a. & G3P 3 & N1nguna & Ninguna & voluntaria & $15 / 43$ & $?$ \\
\hline
\end{tabular}

parto o del aborto las edades oscilaban entre 27 y 32 años, con un promedio de 29.5 años.

Cuatro $(66.6 \%)$ de estas pacientes. presentaron patología obstétrica o puerperal: $2(33.3 \%)$ fueron sometidas a cesárea por desproporción pélvico-fetal, una $(16.6 \%)$ presentó toxemia y tuvo un mortinato, y una $(16.6 \%)$ requirió transfusión de un litro de sangre después de un legrado por un aborto de $\mathbf{4 0}$ días.

De las 4 pacientes con hijos vivos, 2 amamantaron y 2 no.

Todas las pacientes presentaron valores de Prolactina normales. Ninguna tenía antecedentes familiares de menopausia precoz. Todas las pacientes ten ían peso adecuado a su talla.

\section{Sindrome de Sheehan (Tablas Nos, 6 y 7 )}

El diagnóstico de $\mathrm{S}$. de Sheehan se basó principalmente en la anamnesis de la clínica que estas pacientes presentaron en el puerperio.

Las- 3 enfermas nos fueron remitidas mucho tiempo después del parto: a los 6,7 y 17 años, por lo que los datos del examen físico y determinaciones hormonales no corresponden al período de instauración y manifestación clínica florida del síndrome.

Todas las pacientes presentaron durante el parto hemorragia aguda con shock hipovolémico, que requirió transfusión. Las manifestaciones clínicas fueron: caida del vello axilar y pubiano, cefalea, astenia, adinamia, e hipotensión en los 3 casos; en $2(66.6 \%)$ hubo una pérdida de peso mayor de $10 \mathrm{~kg}$. , y en una un aumento superior a los $10 \mathrm{~kg}$. Hubo agalactia en los 3 casos.

La primera paciente fue diagnósticada clínicamente de Síndrome de Sheehan durante el puerperio y tratada inmediatamente $y$ durante 7 años con glucocorticoides y extractos tiroideos; $y$ en el momento de la primera consulta presentaba un aspecto mixedematoso. Se suprimió toda medicación y se practicaron determinaciones hormonales que figuran en la tabla No. 7. La PRL, FSH y LH eran normales. Los valores de T3 y T4, y la curva de captación de I radiactivo evidenciaron una hipofunción tiroidea. El test de TRH fue (-). Las cifras de cortisol $y$ el test de ACTH mostraban un 
Tabla No. 6

\section{SINDROME DE SHEEHAN \\ CUADRO CLINICO}

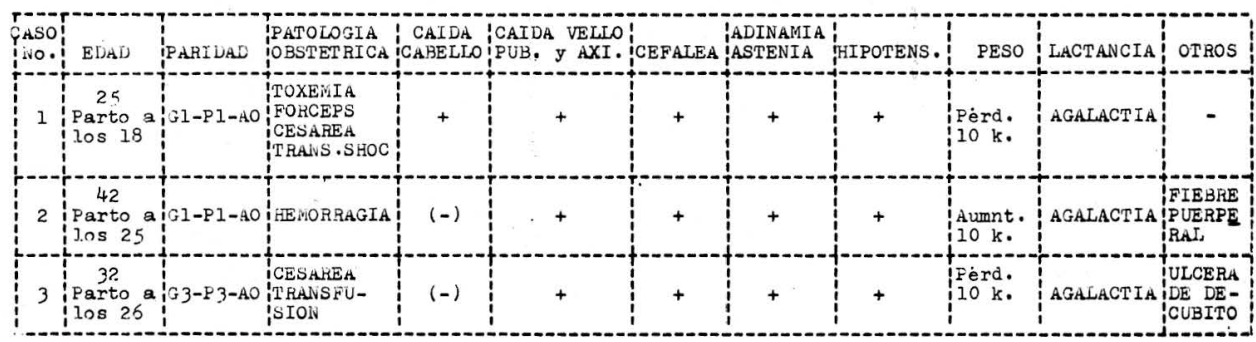

Tabla No. 7

\section{SINDROME DE SHEEHAN \\ DATOS DE LABORATORIO}

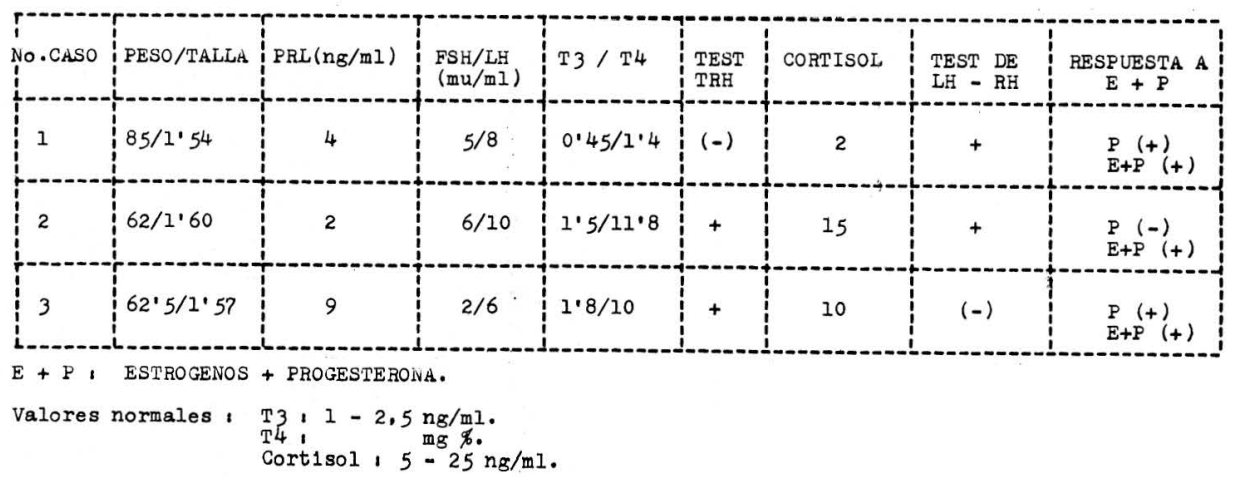

hipocorticismo moderado. Esta paciente fue tratada con dosis adecuadas de extracto tiroideo y supresión de los corticoides, con lo que desapareció la sintomatología hipotiroidea, recuperó su peso normal y presentó varias reglas espontáneas.

Los casos 2 y 3 no recibieron ningún tratamiento en el período que transcu- rrió entre la instauración del cuadro y el momento de consulta, excepto estrógenos y progesterona para provocar hemorragias por deprivación. Los valores de PRL, FSH, LH, T3, T4, Cortisol, y el test de TRH fueron normales.

En las 3 pacientes se practicó test de $\mathrm{LH}-\mathrm{RH}$; en 2 fue positivo $\mathrm{y}$ en una fue negativo. 


\section{Síndrome de Asherman (Tabla No. 8)}

El diagnóstico de estos casos se basó principalmente en los hallazgos histerosalpingográficos. Se practicó una histerosalpingografía siempre que la administración de estrógenos y progesterona fue incapaz de provocar una regla o cuando ésta fue muy escasa.
Las edades de estas pacientes oscilaron entre 27 y 32 años, con un promedio de 29.5 años. Una de ellas $(50 \%)$ era primigestante y la otra $(50 \%)$ multigestante. Las dos $(100 \%)$ fueron sometidas a cesárea, una por sufrimiento fetal y la segunda por placenta previa. Las dos presentaron patología puerperal: una presentó ileo paralííico, absceso de pared

(Tabla No. 8)

\section{SINDROME DE ASHERMAN}

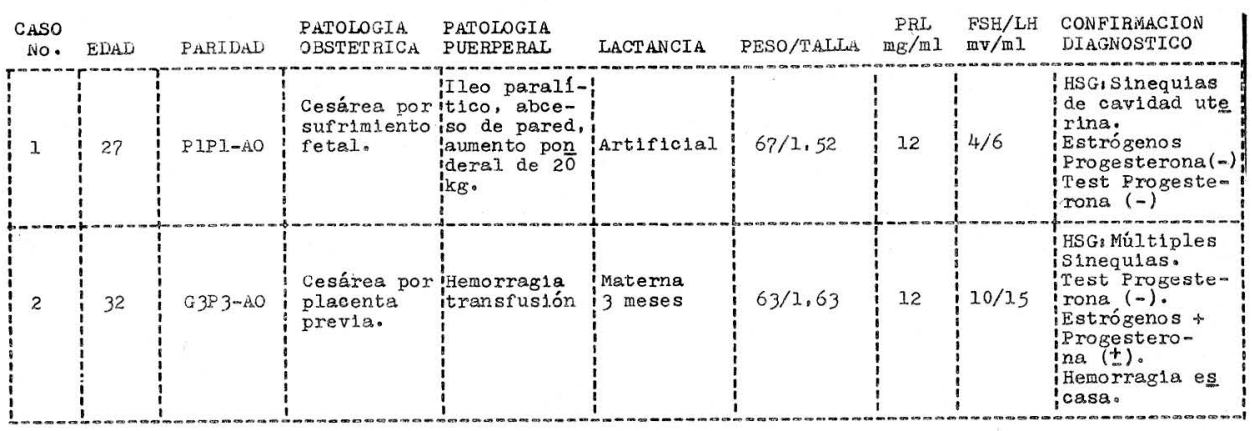

(Tabla No. 9)

PACIENTES CON PATOLOGIA OBSTETRICA Y PUERPERAL.

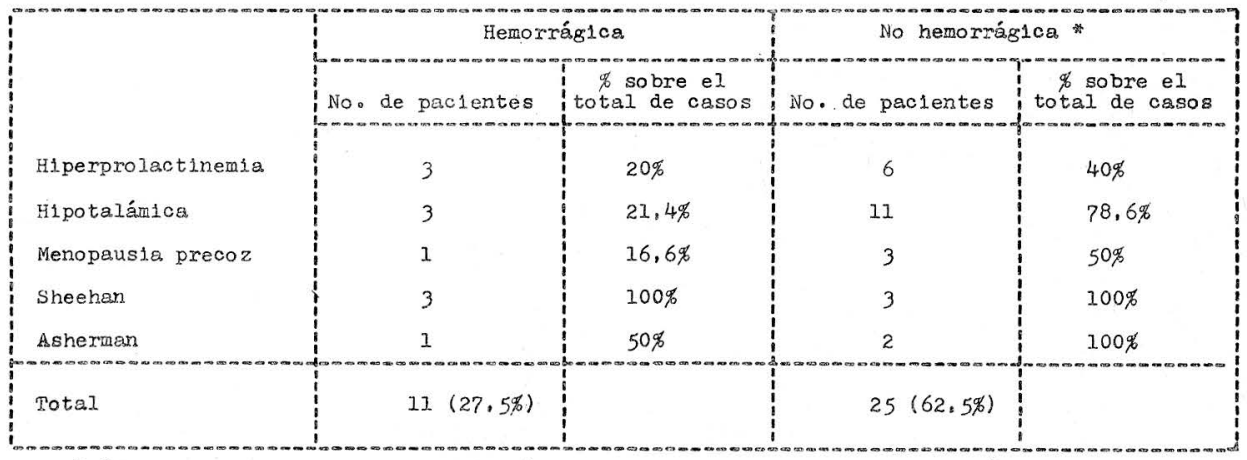

- Inclufdas variaciones ponderales patológicas。 
y aumento ponderal de $20 \mathrm{~kg}$; y la otra presentó una hemorragia que requirió transfusión en el puerperio inmediato.

Una de ellas amamantó y la otra no.

Las determinaciones hormonales fueron normales en ambas pacientes. $Y$ las dos tenían pesos adecuados a su talla en el momento de la consulta.

Correlación entre Patología Obstétrica o puerperal y amenorrea (Tabla No. 9)

De las 40 pacientes, $36(90 \%)$ presentaron algún tipo de patología durante el embarazo parto puerperio. Once (27. $5 \%$ ) presentaron hemorragia moderada o severa; sin embargo, sólo 3 de ellas presentaron Síndrome de Sheehan (27. $2 \%$, y las otras 8 estuvieron repartidas entre amenorreas hipotalámicas: 3 (27. $2 \%)$, hiperprolactinemia: $3(27.2 \%)$, menopausia precoz: $1(9.1 \%)$, y Síndrome de Asherman: $1(9.1 \%)$.

\section{COMENTARIOS}

Múltiples factores pueden ser capaces de provocar una amenorrea después de una gestación. Hay algunos fisiológicos, como la lactancia, y otros patológicos de pronósticos tan distinto como amenorreas de origen hipotalámico funcional, amenorreas hiperprolactinémicas de origen funcional o tumoral, las insuficiencias ováricas precoces o los síndromes de Asherman y Sheehan.

La lactancia aún prolongada durante un año, solo provoca amenorrea persistente en el $40 \%$ de los casos (28). Se considera patológica la amenorrea que persiste más de 3 meses después del parto o de la suspensión de la lactancia (39.13). Nosotros hemos sido más amplios en ese criterio $y$ hemos recogido solamente aquellas pacientes que presentaban una amenorrea mayor de 6 meses. Al clasificar nuestras pacientes en los distintos cuadros cl ínicos etiológicos nos hemos encontrado con una incidencia similar a la reportada por otros autores $(38,70)$.

\section{FORMAS CLINICAS}

\section{Amenorrea hiperprolactinémica}

Se sabe que en el curso del embarazo y debido al aumento progresivo de los estrógenos, hay una hipertrofia del lóbulo anterior de la hipófisis a expensas principalmente de las células lactotropas, que se inicia en el segundo mes y es máxima durante el tercer trimestre. Paralelamente, se produce un aumento de los valores de Prolactina que al final del embarazo son 8 veces mayores que los valores normales $(12,13,16,17,30,31$, $35,40,41,46,56,65,66)$. En el post parto los lactotropos persisten reunidos en cordones, pero su talla se reduce y están casi enteramente vacíos de material granuloso. Los valores de Prolactina vuelven a ser normales entre la $2 a$. y la 6 a. semana post parto. La disminución de los valores basales se produce tanto en las pacientes que amamantan como en las que no, pero en las primeras la succión provoca picos de Prolactina que van disminuyendo en amplitud a medida que se prolonga la duración de la lactancia $(16,17,35,55,65,66)$.

En la primera semana post parto existe una hipogonadotropinemia, los valores de $\mathrm{FSH}$ se van normalizando hacia los 12 a 18 días, tanto si la paciente amamanta como si no, el aumento de la LH es más tardio y menor en las pacientes que amamantan, lo que provoca una mayor incidencia de anovulación en los primeros ciclos post parto $(16,17,35,55)$. En el post parto el ovario se hace refractario a la acción de las gonadotropinas tanto exógenas como endógenas, al parecer porque la prolactina bloquea los receptores hormonales ováricos y altera la modulación de gonadotropinas a nivel hipotalámico $(5,10,15,16,17,28,29$, $34,35,39,43,55,67,70,73)$. 
La hipertrofia causada por los estrógenos en la hipofisis puede persistir durante el post-parto produciendo una hiperprolactinemia, bien de tipo funcional, bien de tipo orgánico, siendo a veces la primera manifestación de adenomas que hasta entonces habian sido asintomáticos $(13,36,39,56,58,64)$.

En 3 de nuestras pacientes, pudimos demostrar la existencia de un adenoma, en otra paciente se sospechaba, y se clasificaron como de tipo funcional los casos en que no pudimos demostrar ningún adenoma.

Aunque Delecour (13) afirma que el hipogonadotropinismo es constante, nosotros sólo lo hemos encontrado en 5 casos $(33.2 \%)$. Sólo en un caso de lactancia materna mayor de un año, encontramos un adenoma. En toda paciente con amenorrea post-gestación hay que buscar la existencia de galactorrea y debe descartarse siempre la presencia de un adenoma hipofisiario (13.22).

En nuestra casuística la galactorrea ha sido espontánea en el $93.3 \%$ de los casos y a la expresión en el $6.7 \%$.

No encontramos ninguna asociación entre los valores más altos de PRL y la existencia de un adenoma. En el $46.7 \%$ de los casos hubo una patología obstétrica o Stress emocional, que han sido descritos como factores desencadenantes de hiperprolactinemia por un mecanismo de alteración de las catecolaminas hipotalámicas $(67,53,39,13,72,50,51,70$, $21,57)$.

El tratamiento con Br-criptina normaliza los ciclos en todos los casos de hiperprolactinemia funcional aunque no lleguen a normalizarse las cifras de prolactina.

\section{Amenorrea hipotalámica}

El que la gestación y el parto o aborto pueden provocar un traumatismo físico o psicológico es evidente (33). Y que la hiperprolactinemia y la disfunción hipotalámica son las principales causas de la amenorrea post parto, también es admitido $(70,38,39,13)$, lo mismo que la asociación entre stress y amenorrea (25, 54), y la localización hipotalámica de los centros del hambre y la saciedad.

Uno de los pilares más importantes para el diagnóstico de estas enfermas es la normalidad de las cifras de gonadotropinas y prolactina; y también las variaciónes del peso, tanto la disminución $(25,14)$, como el aumento $(24,13,33)$. Estas variaciones suelen relacionarse con desórdenes psicoafectivos; se habla incluso de una entidad cl ínica "obesidad o delgadez neurógena del post parto". (13). Parece ser ligeramente superior la incidencia de amenorrea hipotalámica en las multíparas $\left(57^{\circ} \%\right)$ que en las primíparas $(42.9 \%)$; al parecer la ganancia ponderal podría ser favorecida por el número de gestaciones. Es frecuente encontrar patología obstétrica $(85.7 \%$ ). En nuestra casuística sólo encontramos aumento de peso en el $50 \%$ de los casos y ningún adelgazamiento.

En el tratamiento de estas pacientes es fundamental la corrección del peso y la psicoterapia, bastando estas simples medidas para que la regla se normalice. Solo en tres casos tuvimos que recurrir al clomifeno para estimular la ovulación.

\section{Menopausia precoz}

Son muchos los trabajos que describen una amenorrea hipergonadotropa después de un parto $(20,37,46,47$, 48). Aunque no hay acuerdo acerca de la edad que debe considerarse como límite para el diagnóstico, hemos considerado menopausias precoces las que ocurrirían antes de los 35 años (3, 11, $46,20)$.

En cuanto al mecanismo de producción del fallo ovárico es muy discutido. 
Según Erickson (19) los receptores hormonales de L.H. y FSH, necesitan la acción de la FSH para producirse, y hacer que el folículo madure. En períodos prolongados de supresión de FSH, como ocurre en la gestación y en la ingestión de anovuladores, pueden disminuir los receptores y llevar a una atresia folicular irreversible o a una ovarioplejía. Sin embargo, se ha hablado también del traumatismo psiquico del parto (20) como factor desencadenante de una atresia y destrucción folicular en situaciones de disfunción hipotalámica hipofisiaria (13), y también se habla de un proceso autoinmune. En nuestros 6 casos hemos encontrado 4 que presentaban patología obstétrica o puerperal.

\section{Síndrome de Sheehan}

Ya descle 1937 (59) se había descrito el hecho de que la hipófisis hipertrofiada al final del embarazo es muy sensible a los cambios hemodinámicos $y$ puede sufrir necrosis y colapso secundarios a estados de shock de la enferma durante $y$ después del parto. También es conocido que la extensión de la necrosis es proporcional a la cantidad de sangre perdida y a la severidad del shock $(60$, $61,62,63)$. Aunque han sido descritos casos de Síndrome de Sheehan después de partos normales (23), la frecuencia de este síndrome va siendo cada vez más rara en la obstetricia moderna debido a una mejor asistencia obstétrica y a una mayor facilidad de transfusión inmediata cuando se presentan pérdidas hemorrágicas. Ninguna de nuestras pacientes había tenido su parto en un centro hospitalario. Todas habían sido atendidas en hos. pitales comarcales.

El Síndrome de Sheehan tiene manifestaciones muy variables: desde la forma completa hasta la paciente que después de haber hecho manifestaciones clínicas durante el puerperio, permanece asintomática.
Nosotros solo encontramos una paciente que en el momento del estudio presentara manifestaciones clínicas y de laboratorio de hipotiroidismo e hipocorticismo suprarrenal, además de la amenorrea. Las demás pacientes sólo presentaron una clínica de astemia, adinamia, caída del vello púbico y axilar, durante el puerperio, y en el momento del examen permanecían amenorreicas $(1,8$, $18,27,32,42,59,60,61,62,63,66)$. Todas presentaron agalactia.

En 2 de nuestras pacientes obtuvimos respuesta al test de $\mathrm{LH}-\mathrm{RH}$ y en una no. Esta respuesta variable al test de LH-RH ha sido descrita ya por varios autores $(1,27,32,68)$. Ninguna de nuestras pacientes quedó embarazada después, aunque se han descrito casos con pronóstico favorable para la gestación (9.42).

\section{Sindrome de Asherman}

Aunque descrito por Fritsch en 1894, solo después de que Asherman (2), en 1948 publicó varios trabajos, se le concedió verdadera importancia. Aunque su incidencia es por fortuna relativamente baja, es posible que sea mayor que la que se acepta, pues ciertos casos que se manifiestan como hipomenorrea pueden no ser diagnosticados. No es sorprendente su presencia como causa de amenorrea post gestación, si se tiene en cuenta que son los legrados puerperales y post aborto y después de ellos las cesáreas las principales causas de este síndrome $(6,7,52)$. La base fundamental para su diagnóstico es la histerosalpingografía (26) que está indicada siempre que no se obtenga una respuesta adecuada a la administración de estrógenos y progesterona.

Las 2 pacientes que encontramos en nuestra casuística habían-sido sometidas a cesáreas. Una de ellas presentó signos sugestivos de endometritis, lo que está descrito como fenómeno coadyuvante 
en la formación de las sinequias (6). La segunda requirió cesárea por una placenta previa que pudo facilitar especialmente la formación de adherencias a nivel del itsmo.

\section{Relación entre patología obstétrica o puerperal y amenorrea}

Llama la atención la gran incidencia de patología obstétrica o puerperal

\section{RESUMEN}

Un porcentaje relativamente alto de amenorreas secundarias $(11 \%)$ puede ser posterior a una gestación.

Toda amenorrea consecutiva a una gestación, de más de 6 meses de duración, es patológica y requiere una valoración exhaustiva y un tratamiento etiológiso adecuado. La anamnesis es fundamental en este estudio, pero no debe basarse solamente en ella el diagnóstico, pues solamente en un porcentaje pequeño de pacientes $(27.2 \%)$ que presenta-
$(90 \%)$ en estas pacientes que desarro. llaron amenorrea post-gestación. Si bien esta relación es explicable en los casos de Síndrome de Sheehan o de Amenorrea Hipotalámica, (habida cuenta del papel que la hemorragia y el stress desempeñan en la fisiopatogenia de uno y otra), es difícil establecer una correlación significativa con los restantes cuadros patológicos, debido a lo pequeño de la muestra.

ron hemorragia o shock durante el parto y cambios ponderales puerperales, la causa de la amenorrea fue el Síndrome de Sheehan. No debe olvidarse tampoco la importancia que la amenorrea hipo. talámica, la hiperprolactinemia y la menopausia precoz tienen como etiologías de esta amenorrea; $y$ el papel preponderante que las determinaciones hormonales juegan en su diagnóstico. Por lo tanto, el estudio racional de las amenorreas post-gestación será hecho con la combinación juiciosa de la Historia Clínica, el examen físico y el estudio endocrinológico.

\section{POST-GESTATION AMENORRHEA}

\section{SUMMARY}

A relatively high percentage of secondary amenorrhea $(11 \%)$ may appear following a gestation.

All cases of amenorrhea of more than six months following a gestation must be considered pathological and require careful evaluation and treatment. Anamnesis is fundamental but the diagnosis mustn't be based only on it, since only in a small porcentage $(27.2 \%)$ of patients presenting hemorrhage or shock

\section{BIBLIOGRAFIA}

1. AONO T., MINAGAWA J,, KINUGASA T., TANIZAWA O., KURACHI K., (1973). Response of pituitary L.H and during delivery and puerperial changes the cause of amenorrhea was Sheehan Syndrome.

It is also important to keep into consideration hypotalamus amenorrhea, hyperprolactinemia and early menopause as etiology in such amenorrhea, and the predominant role played by hormones in diagnosis. Therefore, rational analyses post-gestation amenorrhea must be based on the medical history, the physical examination and the endocrinological study.
FSH to synthetic LH -releasing-hormo ne in normal subjects and patients with Sheehan's Syndrome. Am. J. Obstet. Gynecol., 117. 1046. 
2. ASHERMAN J. G. (1948). Amenorrhea traumática (atrética). J. Obst. Gynec. Br. Emp., 55, 23.

3. BOARD J., REDWINE F., MONCURE C., FRABLE W., TAYLOR J., (1979). Identification of differing etiologies in clinical diagnosed premature menopause. Am. J. Obstet Gynecol., 134, 936.

4. BONILLA F. (1961). Diagnóstico y Tra tamiento del Síndrome de Sheehan. $C_{0}$ municaciones al III Congreso Mundial de Ginecología, Viena. Edit Congress print Romayor Viena 1961. 1, 14.

5. BONTE M., KINGONEYE A., GASHAKANBA $E$, et al (1974). Malnutrition and post partum amenorrhea. Int. J. Fertil. $19,97$.

6. CARMICHAEL E. (1970). Asherman's Syndrome Obstet. Gynec., 36, 922.

7. COMINOS A., PANTELIS Z. (1969), Treatment of uterine adhesions. Am. J. Obstet Gynecol. 105, 862.

8. COOK J.E., BEAN W., FRANKLIN M., EMRHICK J. (1951). Post partum necrosis of the anterior lobe of pituitary gland. Arch. Intern. Med. 87, 517.

9. COHEN S., BAILLIE P. (1980). Sheehan's Syndrome followed by successful pregnancy، A case report. S. Afr. Med. Journal, 57, 20.

10. CORBEY R., LEQUIN R., ROLLAND R. (1977). Hyperprolactinemia and secondary amenorrhea. Prolactin and human reproduction. Academic Press. London, 1977, 11, 203.

11. COULAN C., RYAN R. (1979). Premature menopause. Am. J. Obstet Gynecol. 133,639 .

12. DAVIES C., JACOBI J., LLOYD $H_{\text {, }}$ MEARES J. (1974). DNA synthesis and the secretion of prolactin and growth hormone by the pituitary gland of the male rats: effects of diethylstill boestrol and $2 \mathrm{Br}$ L. ergocriptine methansulphonate. J.Endocrinol, 61, 411.

13. DELECOUR M., FOSSATI P., CAPPOEN J. (1975). Syndromes neuroendocriniens du post partum, Enciclop. Med. Chir. 5114, G 10, 6 .

14. DELGADO H, BRINEMAN E., LECHTIG A., BONGAARTSY, MARTORELL R., KLEIN R. (1979). Effect of maternal nutritional status and infant supplementation during lactation on post partum amenorrhea. Am. J. Obstet. Gynecol., $135,303$.

15. DEL POZO E., VARGA L., SCHULTZ K., KUNSING H., MARBACH P., EPPENBERGER V. (1975). Pituitary and ovarian response patterns to stimulation in the post partum and in galactorrhea amenorrhea. Obstet. Gynecol. 46, 539.

16. DELVOYE P., BADWI M., DEMAEGD M., ROBYN C. (1978). Long lasting lactation is associated with hyperprolactinemia and amenorrhea en: ROBYN C., HARTER M. Progress in prolactin physiology and pathology. Editorial Biomedical press Amsterdam 1978. pág. 128.

17. DELVOYE P., DEMAEGD M., NYAMPETA V., ROBYN C. (1978). Serum prolactin, gonadotropins and estradiol in menstruating and amenorrheic mothers during two year's lactation. Am. J. Obstet Gynecol. 130, 635.

18. DIZERAGA G., KLETZKY O., MISHELL D. (1978). Diagnosis of Sheehan's Syndrome using a sequential pituitary stimulation test. Am. J. Obstet Gynecol. 132, 348.

19. ERICKSON G.F. (1978). Normal ovarian function. Clin. Obstet. Gynecol. 21, 31, 1978.

20. FERRER-BARRIENDOS J., MILLET A. (1978). Menopausia precoz. Revisión de conjunto. Rev. Esp. Obst. Gin. 37, 211. 
21. FOSS G.L., SHORT D. (1951). Abnormal lactation. Journal. Obst. Gynecol. Br. Emp. 48, 35.

22. FOSSATI M., ASFOUR, CORTIER J.C. (1976). Hypopituitarisme antérieur et global du post partum. Rev. franc. Gynéc. 71, 179 .

23. FOSSATI C., KHERN A., DUBECQ J. CLAPIES J., GONNET J., (1979). Syndrome de Sheehan aprés accouchement normal. J. Gyn. Obst. Biol. Repr. 8, 379.

24. FISHMAN J., BOYAR R., HELLMAN L, (1975). Influence of body weight on estradiol metabolism in young women. J. Clin. Endocrinol. Metab. 41, 989.

25. FRIES H., NILLIUS, PETTERSON $F$. (1974). Epidemiology of secondary amenorrhea. A retrospective evaluation of etiology with special regard to psychogenic factors and weight loss. Am. J. Obstet. Gynecol. 118, 473.

26. HALD H. (1949). On uterine atresia consequent to curettage. Acta Obst. Gynecol. Scand. 28, 169.

27. HADDOCK L., VEGA L., AGUILO F. RODRIGUEZ O. (1972). Adrenocortical thyroid and human growth hormone re serve in Sheehan's Syndrome. John Hopkins Med. J. 131, 80.

28. HEFNANI F., ISMAIL H., YOONIS N., et al (1977). The benefit of lactation amenorrhea as a contraceptive. Egy-Int. J. Gynecol. Obstet 15, 60.

29. HOYUEN B., CANNON W., $S$ y L., BOO TH J., BURGH P. (1982). Regression of pituitary microadenoma during and follo wing bromocriptine therapy. Persistent defect in prolactin regulation througho ut pregnancy. Am. J. Obstet Gynecol. $142,634$.

30. HUNT T.E. (1947). Mitotic activity in the anterior hypophysis of ovariectomized rats after infection of estrogens. Anatomical Record 97, 127.

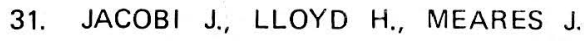
(1977). Onset of oestrogen-induced prolactin secretion and D.N.A. synthesis by antherior pituitary gland. J, Endocrinol $72,35$.

32. JACKSON I.M., WHYTE D., GARREY M. (1969). Pituitary function following un complicated pregnacy in Sheehan's Syn drome. J. Clin. Endocrinal. 29, 315.

33. LAC HELIN G., YEN S.C. (1978). Hypothalamic cronic anovulation. Am. J. Obstet Gynecol. 130, 825.

34. L'HERMITE M., VEKEMANS M., CAUFRIEZ A., MARIN COMIN J., ROBYN C. (1975). Effect of sulpiride induced hyperprolactinemia on human menstrual cycle. Acta. Endocr. (kbh) 80, suppl. $199,322$.

35. L'HERMITE M. (1976). Fonctions gonadotrope et prolactinique au cours de la grossesse et du post partum. Rev.franc. Gynéc. 71, 159.

36 LE POGAMP C., GRALL J.Y., MASSART C., RAMEE A., TOULOUSE R. (1979). Poussée évolutive $d^{\prime}$ adenome a prolactine au cours d'une grossesse aprés sterilité traitée, 2 observations. Nouv. presse Med. 8, 2009.

37. LE POGAMP, KERISIT J., MASSART C., MENTION J.E. (1980). Aménorrhée ovarioplégique. J. Gyn. Obst. Biol. Repr. $9,771$.

38. LE POGAMP C., MASSART C., GRALL J. (1981). Place de la contraception orale et de la grossesse dans les étiologies des aménorrhées. J. Gyn. Obst. Bio. Repr. $10,223$.

39. LINQUETTE M. (1976). Les syndromes aménorrhée-galactorrhée du post-partum. Rev. Franc. Gynéc. 71, 173.

40. LLOYD A., MEARES J., JACOBI J. (1973). Early effects of stilboestrol on growth hormone and prolactin secretion and on pituitary mitotic activity in the male rat. J. Endocrinol 58, 227. 
41. LLOYD H., MEARES J., JACOBI J. (1975). Effects of oestrogen and bromo cryptine on in vivo secretion and mitosis in prolactin cells. Nature 225, 497.

42. MARTIN J., KAPLAN N. (1970). Successful pregnancy in a patient with Sheehan's Syndrome, New. Eng. J. Med. 282, 45.

43. Mc NATTY K.P., SAWERS R.S., Mc NEILLY A.S. (1974). A possible role of prolactin in centrol of steroid secretion by the human graafian follicle. Nature (Lond) 250, 653,

44. MEITES J., NICOLL C.S. (1966). Adenohypophysis: prolactin. Ann. Rev. Physiology 28,57 .

45. MILLET A, F. GIL GRACIA (1977). Valores normales de FSH y L.H a lo largo del ciclo femenino. Rev. Esp. Obst Gin. 36,285 .

46. MHLLET A., FERRER-BARRIENDOS. J., MORENO J. (1978). Menopausia precoz. Experiencia clínica a propósito, 30 casos. Rev. Esp. Obst. Gin. 37, 223.

47. NETTER A, SEBAOUN M. (1966). Menopause précoce. Etude anatomique, et chromosomique de 16 cases dont deux mosaiques. Gynec. et Obstet. 66, 249,

48. NETTER A., CARMEN G., ROZEMBAUM H, (1978). Le syndrome des ovaries resistants aux gonadotrophines. Actualites Gynécologiques géne série. Edit. Masson París. 1978. pg. 24.

49. MUEHLENSTEDT D. (1981). Hypophyse und schwangerschaft. Aktuel endo krinol stoffwechsel. 2, 53.

50. NICOLL C.S., TALWALKER P.K., MEITES J. (1960). Initiation of lactation in rate by nonespecific stresses. Am. J. Physiol. 198, 1103.

51. NOEL G., SUH H., STONE J. et al. (1972). Human prolactin and growth homone release during surgery and other conditions of stress. J. Clin. Endocrinol. Metab. 35, 840.

52. ONETO E., SAAVEDRA R., CRISOSTOS C. (1965). The treatment of uterine synechiae with the help of iatrogenic pseudo-pregnancy. Int. J. Fertil. 10, 217.

53. QUIGLEY M., YEN S. (1980). Evidence of increased dopaminergic inhibition of secretion thyroid-stimulating hormone in hyperprolactinemic patients with pituitary microadenoma. Am. J. Obstet. Gynecol. 137. 653.

54. RABKINS J.G., STRUENING E. (1976). Life events stress and illness. Science. 194, 1013.

55. REYES F., WINTER J., FAIMAN C. (1972). Pituitary-ovarian in interrelationships during the puerperium. Am. J. Obstet. Gynecol. 114, 589.

56. ROWE T., SHEARMAN R., FRASER J. (1979). Antecedent factors and outcome in amenorrhea-galactorrhea. Obstetrics and Gy necology 54, 535.

57. SACHAR E., FRANTZ A., ALTMANN, SASSIN J. (1973). Growth hormone and prolactin in unipolar and bipolar depres sed patients: Response to hypoglucemia and L-DOPA. Am. J. Psychiatr. 130, 12.

58. SHEARMAN B., SCHELECHTE J., HAL. MI N. (1978). Pathogenesis of prolactinsecreting pituitary adenomas. Lancet, 2, 1019.

59. SHEEHAN H.L. (1937). Post-partum ne crosis of anterior pituitary. J. Path. Bact. 45, 189.

60. SHEEHAN H.L. (1939). Simmond's di sease due to post-partum necrosis of the anterior pituitary. Q.J. Medical. 8. 277.

61. SHEEHAN H.L., SUMMERS V.K. (1949) The syndrome of hypopituitarism. Q.J. Medical. 18, 319.

62. SHEEHAN H.L. (1954). The incidence of post-partum hypopituitarism. Am.J. Obstet. Gynecol. 68, 202. 
63. SHEEHAN H.L. (1965). The frequency of post-partum hypopituitarism. J. Obstet. Gynecol. Brt. Cwlth. 72. 103.

64. SHENCHUCK A., ADAMSON D., LES SARD P., EZRIN C. (1980). The eftect of pregnancy on suspected pituitary adenomas after conservative management of ovulation defects associated with galactorrhea. Am. J. Obstet. Gynecol. 136. 659.

65. TYSON J.E., AWANG P., GUYDA H., FRIESEN H.G. (1972). Studies of prolactin secretion in human pregnancy. Am. J. Obstet. Gynecol. 113, 14.

66. TYSON J.E., KHOJANDI M., ANDREASSEN B. (1975). The influence of prolactin secretion on human lactation. J. Clin. Endocrinol. Metab. 40. 764.

67. TYSON J.E., ANDREASSON B., HUTH J., SMITH B., ZACUR A. (1975). Neuroendocrine dysfunction in galactorrheaamenorrhea after oral contraceptive use. Obst. Gynec. 46, 1.

68. VALCKE J., MAHODEAU J., THIEBLOT Ph., PIQUEL, et al (1974). Criteres $\mathrm{d}^{\prime}$ interpretacion $\mathrm{du}$ test utilisant
I'hormone de liberátion de la luteostimuline. Ann. Endocr. (Paris) 3, 423.

69. VAN LOON G.R. (1978). A deffect in cathecolamine neurons in patients with prolactin-secreting pituitary adenoma. Lancet 2, 868.

70. VAN LOONP.F., MCNEILLY A.S., HUNTER W.M., BAIRD D.T. (1977). The role of prolactin in secondary amenorrhea. Proceeding of the serono symposia. Academic Press. London 1977. 11, 17.

71. VEZINA J., SUTTON T. (1974). Prolactin secreting pituitary microadenoma. Amer. J. Roentgenol. 120. 46.

72. YLIKORKALA O., RONNEBER L. (1980). Prolactin changes in maternal plasma following termination by vacuum curettage and the effect of $\mathrm{Br}$. criptine treatment of these changes. Brit J. Obstet Gynecol 87, 911 .

73. ZARATE A., CANALES E., SORIA J., RUIZ F., McGREGOR C. (1972). Ovarian refractoriness during lactation in women: Effect of gonadoproph in stimulation. Am. J. Obstet Gynecol. 112, 1130. 